\title{
ON THE PROCESS OF FLAME SPREADING OVER THE SURFACE OF PLASTIC FUELS IN AN OXIDIZING ATMOSPHERE
}

\author{
CARLOS SANCHEZ TARIFA, PEDRO PEREZ DEL NOTARIO, \\ AND ANTONIO MUÑOZ TORRALBO \\ Instituto Nacional de Técnica Aeroespacial, Madrid, Spain
}

\begin{abstract}
A general theory of flame spreading over the surface of solid or liquid fuels in an oxidizing atmosphere is developed in the present work.

The model of the process considers heating of the fuel ahead of the flame, fuel vaporization and mixing with the gaseous oxidizer and flame propagation through this combustible mixture. The flame spreading velocity restults from the balance of all these processes.

This theory is of a general type and may be applied to many kinds of flame spreading processes. However, the work has been directed to the case of flame spreading over the surface of plastic fuels in nitrogen-oxygen mixtures.

The model of the process is solved by approximate analytical methods and an experimental investigation is also carried out. Theoretical and experimental results are obtained and compared, showing the influence of initial fuel temperature, pressure and mixture composition.
\end{abstract}

\section{Introduction}

A complete analysis of the general problem of flame spreading along the interface between a solid or liquid fuel and a gaseous atmosphere which can react with the fuel vapors has not yet been carried out although a large number of studies, mostly experimental, have been performed on several aspects of the problem, especially in connection with fire safety regulations and fire spreading in air..$^{1-4}$ Flame spreading processes have also been studied in rocket motors, but most of these studies refer to the case of solid monopropellants, which is a problem of a different nature. On the other hand, very little information exists on flame spreading processes over the surface of solid propellants in a reactant atmosphere in connection with ignition processes in hybrid rockets. ${ }^{5,6}$

A thermal theory of flame spreading along the surface dividing a solid or liquid fuel from an oxidizing atmosphere was developed by the authors of the present work. ${ }^{6}$

This theory was essentially based on the existence of an ignition temperature, and the flame spreading velocity was calculated on the assumption that the flame propagates to a certain location when the fuel surface temperature at that location reaches an ignition temperature.

The introduction of the concept of the ignition temperature permits a simple study of the process. However, since the ignition temper- ature is not a real physico-chemical property of the process, its value is uncertain because it depends on the ignition procedure. Furthermore, the influence of very important variables of the process, such as the pressure and composition of the gaseous atmosphere, cannot be calculated theoretically. Another additional difficulty is that spreading velocities cannot be studied for initial temperatures of the fuel equal to, or higher than, the ignition temperature.

A more general theory of flame spreading is developed in the present work. The concept of ignition temperature has been eliminated and all the essential processes which are part of the over-all flame spreading process are taken into account: heating of the fuel by the flame, fuel vaporization, mixing of the fuel vapors with the gaseous atmosphere and flame propagation through this combustible mixture; the flame spreading velocity results from the balance of all these processes. The over-all process is extremely complex because of the complicated nature of the partial processes, and no information is available on the values of many important parameters, especially in connection with evaporation laws and chemical kinetics of combustion. Therefore, the scope of the present work will be limited to the development of a general model of the over-all process which will permit a qualitative study of the spreading velocity. This model will also permit the analysis of the influence on this velocity of each partial process as well as that of the main variables of all these 


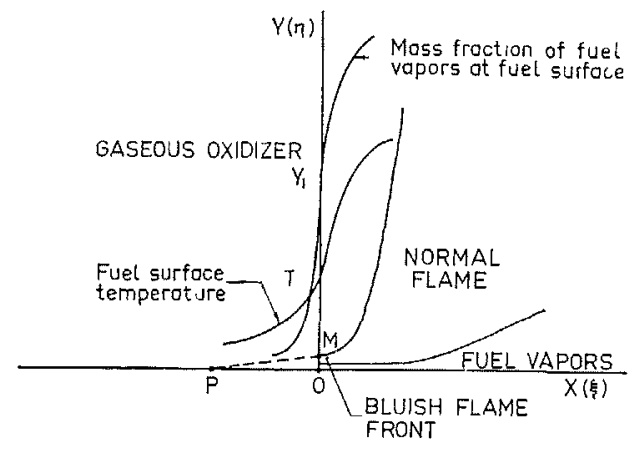

SOLID FUEL

Fig 1 Flame plopagation model

processes The theor etrcal model of the spreading process will be of a very general type However, the wolk will be dnected to the case of flame spreading over the surface of plastic fuels in nitrogen-oyygen mixtures, on which an experimental progiam has also been canred out in older to have a qualitative companison between theoretical and experimental results

\section{Fundamental Assumptions}

21 The following fundamental assumptions have been made Conditions are stationaly with respect to a coordinate system moving with the flame, chemical reactions take place in the gas phase, and there is bidimensional symmetry The flame spreading process results from heating and vaporization of the fuel, mring of the fuel vapors with the gaseous oxidizer and flame propagation through this combustible mixture

Staiting with an assumed value $u$ of the spreading velocity and using approumate explessions for the heat fluxes from the flame to the fuel, the temperature distribution at the fuel surface $T_{s}=f_{1}(r, u)$ can be calculated With these temperatures and using a vapoization law, diffusion equations give the fuel vapor mass fraction at the fuel sufface, $Y_{1 s}=f_{2}(x, u)$ Finally, the flame propagation velocity can be calculated as function of $Y_{1 s}$, and by equating the mitial and final velocities at $\imath=0$, the actual spreading velocity is obtained

The study of all these processes will be based on several assumptions which are discussed in the following paragraphs

\section{Heating of the Fuel}

Heating of the fuel ahead of the flame may take place through 1adiation, conduction of convection A point $P$ at the fuel surface ahead of the flame will be heated duectly by ladiation, and by heat conduction through the gaseous atmosphere It can also be heated through convection and iadiation fiom the flame to the fuel and then by heat conduction within the fuel, following a path such as MOP ( $\mathrm{F}_{1 \mathrm{~g}} 1$ ) Heat conduction through the gaseous atmosphere predommates over heat conduction within the fuel, if the theimal diffusivity $\lambda / \rho c_{p}$ of the gas is laiger than the theimal diffusivity of the fuel This is the case, at ambient pressure, for $\mathrm{N}_{2}-\mathrm{O}_{2}$ mixtures and plastic fuels, but the opposite occurs at high pressure or for other materials (Table I)

The radiant heat flus reaching the fuel surface ahead of the flame may be approsimated by an expression of the form

$$
Q_{R}(\imath)=\epsilon \sigma T_{F}^{4} T^{\prime}
$$

in which $F$ is the geometical factor, which may be approsmated by an exponential function of the distance from the flame front The flame emissivity $\epsilon$ depends exponentially on the flame size

Heat transfelıed by convection to the fuel suface may be calculated by a one-dimensional

TABLE I

Approximate values of theimal diffusivities at ambient temperature

\begin{tabular}{|c|c|c|c|c|c|c|}
\hline & \multicolumn{2}{|c|}{ Mratiues $\mathrm{N}_{2}-\mathrm{O}_{2}$} & \multirow[b]{2}{*}{ Plexıglass } & \multirow[b]{2}{*}{$n$-Heptane } & \multirow[b]{2}{*}{ Magnesium } & \multirow[b]{2}{*}{ Alumminm } \\
\hline & $\begin{array}{c}p=1 \\
\text { atm }\end{array}$ & $\begin{array}{c}p=300 \\
\text { atm }\end{array}$ & & & & \\
\hline $\begin{array}{c}\lambda / \rho c_{p} \mathrm{~cm}^{2} \\
{ }^{\circ} \mathrm{C} / \mathrm{sec}\end{array}$ & $\sim 025$ & $\sim 08 \times 10^{-3}$ & $\sim 12 \times 10^{-3}$ & $\sim 09 \times 10^{-3}$ & $\sim 19$ & $\sim 45$ \\
\hline
\end{tabular}


approximation, but in the present work it will be assumed, for sumplicity, that radiant heat transfer is predominant as compared with heat conduction, as usually occurs provided that the flame is not too small.

Convection and radiative heat transfer behind the flame fiont will not influence the process tor the present case of a plastic fuel.

Once the expression of the heat flux is given, heat conduction within the fuel can be calculated by an approximate method based on the assumption that heat transfer parallel to the fuel surface is small as compared with heat transfer perpendicular to the fuel surface.

\subsection{Fuel Vaporization and Mixing}

Fuel vaporization as function of the fuel surface temperature will be assumed to be given by a Langmuir's expression of the type

$$
\dot{m}_{f s} / \rho_{1} \nu=K_{1} \exp \left(-\frac{q_{1} / R}{T_{s}-T_{\infty}}\right) .
$$

Diffusion of vapors into the oxidizer is calculated, neglecting both convective transport perpendicular to the fuel surface and diffusion parallel to the fuel surface. Free convection effects are not considered.

\subsection{Flame Propagation}

It may be observed that the flame spreads over the surface of a plastic fuel as a thin bluish flame front which moves very close to the fuel surface. Combustion in this thin advancing front probably takes place at fuel-oxidizer ratios very close to the minimum value compatible with flame propagation. Behind this thin advancing flame, we find the brilliant and largest part of the flame, where combustion probably is independent of the conditions existing in the advancing flame front. Therefore, it is reasonable to assume that the spreading process is controlled by the mixture conditions at the fuel surface, and to calculate the propagation velocity for the onedimensional case, with the mixture conditions existing at the flame front. It may be pointed out that the normal part of the flame will be responsible for the radiant heat transmitted, which will not depend on the local conditions existing at the advancing flame front.

\section{Mathematical Study of the Process}

3.1 The mathematical treatment of all the partial processes has been simplified considerably in order to permit analytical study of the com- plete process. Nevertheless, numerical methods may be applied using the physical model developed in the present work.

\subsection{Temperature at the Fuel Surface}

The heat transport equation within the fuel is:

$$
\begin{aligned}
\left(\partial^{2} T / \partial x^{2}\right)+\left(\partial^{2} T / \partial y^{2}\right) & \\
& -\left(\rho_{f} c_{f} u / \lambda_{f}\right)(\partial T / \partial x)=0 .
\end{aligned}
$$

In Ref. 6, this equation was solved by an integral method. In this paper, it will be assumed that the product of the Reynolds and Prandtl numbers:

$$
\operatorname{Re} \operatorname{Pr}=\rho_{f} c_{f} u L / \lambda_{f}
$$

is large. In this expression $L$ is a characteristic length, such as $\delta_{f}$, for which the heat flux reaching the fuel surface is significant. (At low spreading velocities the approximation of this simplification is obviously questionable.)

If it is also assumed that the thickness of the thermal boundary layer in the fuel is small as compared with $L$, then Eq. (3), in dimensionless coordinates, reduces to:

$$
\partial^{2} \theta / \theta \eta^{2}=(u / \nu)(\partial \theta / \partial \xi)
$$

where $u$ is the flame spreading velocity and $\nu$ a characteristic velocity of the process.

With the above assumptions, neglecting the heat transmitted by the fuel surface and taking as expression for the radiant heat flux reaching the fuel:

$$
Q(x)=Q_{R} \exp \left[\beta\left(x / \delta_{f}\right)\right]=Q_{R} \exp (\beta \xi)
$$

the boundary conditions reduce to:

$$
\begin{array}{cc}
\xi=-\infty & \theta=\theta_{\infty} \\
\eta=-\infty & \theta=\theta_{\infty} \\
(\partial \theta / \partial \eta)_{\eta=0}=\exp (\beta \xi) .
\end{array}
$$

Integrating Eq. (5) for the above boundary conditions, we obtain for the temperature distribution at the fuel surface:

$$
\theta_{s}=\theta_{\infty}+\exp (\beta \xi)[\beta(u / \nu)]^{-1 / 2} .
$$

\subsection{Mixing of the Fuel Vapor with the Oxidizer}

Assuming that the diffusion coefficient and densities are constants, mixing of the fuel vapors 
will be controlled by the equation:

$$
\begin{aligned}
V_{x}\left(\partial Y_{1} / \partial x\right)+V_{y}\left(\partial Y_{1} / \partial y\right) & =D\left(\partial^{2} Y_{1} / \partial x^{2}\right) \\
+ & D\left(\partial^{2} Y_{1} / \partial y^{2}\right) .
\end{aligned}
$$

The vaporization rate is very small, therefore, the term $V_{y} \partial Y_{1} / \partial y$ will also be very small. Assuming also that the product of the Reynolds and Schmidt numbers:

$$
\operatorname{ReSc}=L V_{x} / D
$$

is large, then diffusion parallel to the fuel surface can be neglected as compared with the convective term. Assuming finally that the thickness of the diffusion boundary layer is small as compared with $L$, Eq. (10) reduces to:

$$
\left(\partial^{2} Y_{1} / \partial \eta^{2}\right)-\left(u \delta_{j} / D\right)\left(\partial Y_{1} / \partial \xi\right)=0
$$

The boundary conditions are:

$$
\begin{array}{cc}
\xi=-\infty & Y_{1}=Y_{1_{\infty}} \\
\eta=\infty & Y_{1}=Y_{1_{\infty}} \\
\left(D / \nu \delta_{f}\right)\left(\partial Y_{1} / \partial \eta\right)_{\eta=0} \simeq-\left(\dot{m}_{f s} / \rho_{1} \nu\right) \\
=-K_{1} \exp \left[-\tau_{0} /\left(\theta_{s}-\theta_{\infty}\right)\right]
\end{array}
$$

in which $\theta_{s}$ is given by Eq. (9).

It will be assumed that the value of $Y_{1_{\infty}}$ in function of the fuel temperature $\theta_{\infty}$, corresponds to the equilibrium conditions and that these conditions are given by an exponential function:

$$
Y_{1_{\infty}}=K_{2} \exp \left[K_{3} /(\Delta-1)\right]
$$

in which $\Delta$ is the ratio of $T_{\infty}$ to a reference temperature $T^{0}$.

Integration of Eq. (12) is not straightforward because of boundary condition (14). It can be achieved approximately by writing the equation in integral form and by expressing the value of $\dot{m}_{f s}$ given by (14) in the form:

$$
\dot{m}_{f s} / \rho_{1} \nu \simeq C_{1} \exp \left(C_{2} \xi\right)
$$

where the values of $C_{1}$ and $C_{2}$ are numerically adjusted according to each initial value of $u / \nu$, in order to approximate the real expression of $\dot{m}_{f s} / \rho_{1} \nu$.

The final solution at the origin is given by:

$$
Y_{1 s 0}=Y_{1 s_{\infty}}+C_{1}\left[B C_{2}(u / \nu)\right]^{-1 / 2} .
$$

Figure 2 shows the curves of $Y_{1 s 0}$ in function of the assumed value of $u / \nu$ for different values of the initial fuel temperature represented by the parameter $\Delta$.

\subsection{Flame Propagation}

\subsubsection{Mathematical Model}

Based on the assumptions of Sec. 2.4, flame propagation in the gaseous phase has been calculated for the one-dimensional case, using the mixture composition at the fuel surface and at the flame front $(\xi=0)$. This mixture consists of oxidizer and fuel vapors, $Y_{1 s 0}$ being the mass fraction of the fuel vapors, as calculated in the preceding paragraph.

The mathematical model of this one-dimensional flame propagation process is based on the assumptions that conditions are laminar and stationary with respect to the flame front. Only two chemical species will be considered: the reactants 12 , formed by a mixture of fuel vapors 1 and oxidizer 2 , and the combustion products 3 . The oxidizer 2 can be a mixture of oxygen and nitrogen or pure oxygen. Constant and equal values of the specific heats and thermal conductivities are assumed for both species.

Finally, an over-all reaction rate of the form:

$$
w_{3}=\Omega \rho^{n}\left(1-Y_{3}\right)^{n} \exp [-(E / R T)]
$$

will be considered.

Under these assumptions, the equations of energy, diffusion and continuity in dimensionless coordinates are given by:

$$
\begin{gathered}
d \tau / d z=\tau-\epsilon_{3} \\
\operatorname{Le}\left(d Y_{\mathrm{z}} / d z\right)=Y_{3}-\epsilon_{\mathrm{B}} \\
d \epsilon_{3} / d z=\Lambda\left(1-Y_{3}\right)^{n} \frac{\exp \left[-\tau_{E} /\left(\tau+\tau_{r}\right)\right]}{\left(\tau+\tau_{r}\right)^{n}}
\end{gathered}
$$

in which the parameter $\Lambda$, given by:

$$
\Lambda=\dot{m}_{12}{ }^{-1} \Omega_{\rho_{0}}{ }^{n} T_{\infty}{ }^{n}\left(\bar{\lambda} / \bar{c}_{p}\right)\left(\bar{c}_{p} / q_{r}\right)^{n}
$$

is the "eigenvalue" of the system.

The boundary conditions are as follows:

$$
\begin{array}{ll}
z=-\infty & Y_{3}=0\left(Y_{12}=1=Y_{1 s 0}+Y_{2 s 0}\right) \\
(\xi=-\infty) & \epsilon_{3}=0 \\
& \tau=0\left(\text { or } \theta=\theta_{\infty}\right) \\
z=+\infty & Y_{3}=1 \\
(\xi \pm+\infty) & \epsilon_{3}=1 \\
& \tau=1
\end{array}
$$




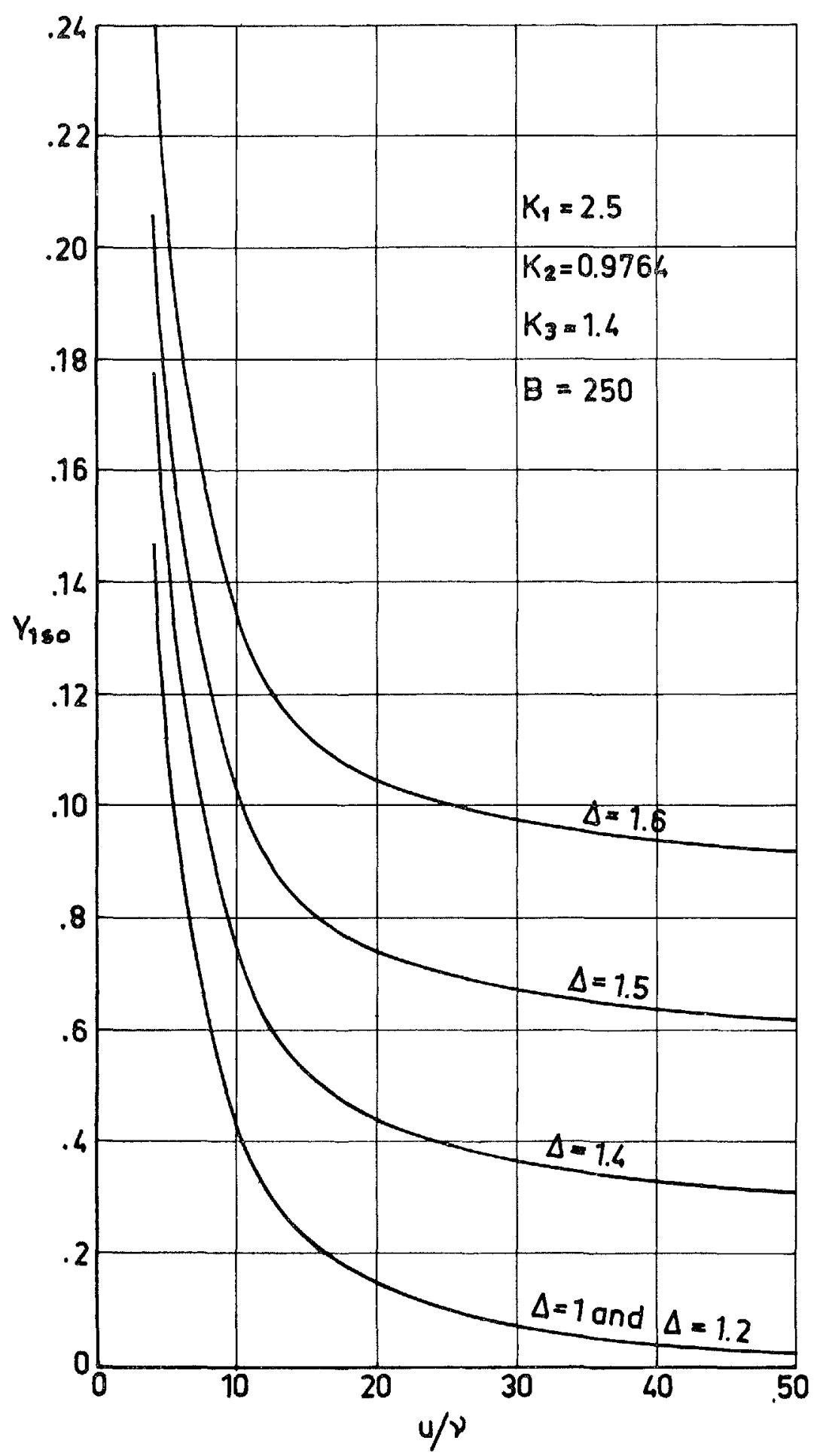

FIG. 2. Fuel vapor mass fraction $Y_{180}$ at the fuel surface and at the origin as a function of the assumed value of the flame spreading velocity, for several values of the fuel temperature at infinity. 
These conditions imply that derivatives $d Y_{3} / d z$, $d \epsilon_{3} / d z$ and $d \tau / d z$ must also be equal to zero for $z= \pm \infty$

All these denvatives, except $d \epsilon_{3} / d z$, are automatically equal to zero for $z=-\infty$ This contradiction at the cold boundary is a well known formal difficulty in theorles of laminar flame propagation In ou case, where the existence of a reaction zone of finite thickness is assumed, that condition is automatically satisfied

\section{Soluthon of the Systern}

Systems (19), (20), and (21) are solved by an approximate analytical method based on the assumption that the reaction zone has a finite thickness $\zeta=z_{\mathrm{II}}-z_{\mathrm{I}}$, and by approaching the flus fiaction $\epsilon_{3}$ within that zone by means of parabolic expressions

A similar method was used in Refs 7 and 8 to study flames with spherical symmetry, and gives an excellent approximation of the process

Fo1 simplicity, it is assumed that the LewsSemenov number is equal to one 'Then, the flux fraction $Y_{3}$ will be equal to the dimensionless temperature $\tau$

Therefore, in the region ahead of the flame $\left(-\infty \leq z \leq z_{1}\right) \epsilon_{3}$ is considered equal to zero, and the flux fraction and temperature ale given by

$$
Y_{3}=\tau=\exp z
$$

Behund the flame $\left(z_{\text {II }} \leq z \leq+\infty\right) \epsilon_{3}$ Is equal to unity and $\tau$ and $Y_{3}$ are also equal to one

Within the flame, by taking

$\epsilon_{a}=-\left(\epsilon_{3}{ }^{* * / 2} / 2 z_{\mathrm{I}}\right)\left(z_{\mathrm{I}}-z\right)^{2} \quad\left(z_{\mathrm{I}} \leq z \leq 0\right)$

and

$\epsilon_{3}=1-\left(\epsilon_{3}{ }^{*} / 2 z_{\mathrm{II}}\right)\left(z_{\mathrm{II}}-z\right)^{2} \quad\left(0 \leq z \leq z_{\mathrm{II}}\right)$

where $\epsilon_{3}{ }^{\prime *}$ is the maximum value of $d \epsilon_{3} / d z$, the following expressions are obtamed for the temperatures and mass fiactions

$$
\begin{array}{r}
\tau=Y_{3}=\exp z+\left(\epsilon_{3}{ }^{*} / z_{\mathrm{I}}\right) \exp \left(z-z_{\mathrm{I}}\right) \\
-\left(\epsilon_{3}{ }^{*} / 2 z_{\mathrm{I}}\right)\left[1+\left(z_{\mathrm{I}}-1-z\right)^{2}\right] \\
\left(z_{\mathrm{I}} \leq z \leq 0\right)
\end{array}
$$

and

$$
\begin{gathered}
\tau=Y_{3}=\left(e_{3}^{\prime *} / z_{\mathrm{II}}\right) \exp \left(z-z_{\mathrm{II}}\right) \\
-\left(e_{3}{ }^{* *} / 2 z_{\mathrm{II}}\right)\left[1+\left(z_{\mathrm{II}}-1-z\right)^{2}\right]+1 \\
\left(0 \leq z \leq z_{\mathrm{II}}\right)
\end{gathered}
$$

By equalizing both values of $\epsilon_{3}$ at $z=0$, we obtain

$$
\left[\left(z_{I I}-z_{I}\right) / 2\right] \epsilon_{3}{ }^{*}=1
$$

The equality of the values of $\epsilon_{3}$ also imples the equality of the delivatives of temperature and mass fiactions at $z=0$

Parametels $\epsilon_{3}{ }^{*}, z_{\mathrm{I}}$, and $z_{\mathrm{I}}$ are obtained by stating that at $z=0$, the value $\epsilon_{3}{ }^{*}$ is given by expiession (21), that is

$\epsilon_{3}{ }^{*}=\Lambda\left(1-Y_{3}^{*}\right)^{n} \frac{\exp \left[-\tau_{\Gamma} /\left(\tau^{*}+\tau_{r}\right)\right]}{\left(\tau^{*}+\tau_{r}\right)^{n}}$

and that at $z=0, \epsilon_{3}{ }^{*}$ is a maximum

$$
\begin{aligned}
-\frac{n Y_{3}^{*}-\epsilon_{3}^{*}}{\operatorname{Le}\left(1-Y_{3}{ }^{*}\right)}+\frac{\tau_{L}\left(\tau^{*}-\epsilon_{3}^{*}\right)}{\left(\tau^{*}+\tau_{r}\right)^{2}} & \\
-\frac{n\left(\tau^{*}-\epsilon_{3}^{*}\right)}{\tau^{*}+\tau_{r}} & =0
\end{aligned}
$$

Finally, by equating the temperatures at $z=0$, the additional condition needed to obtain the eigenvalue $\Lambda$ of the system is obtamed This eigenvalue gives the mass flow $m_{12}$ per unit area, and from it the spreading velocity is calculated

From (26) and (27), we obtam

$\frac{\exp \left(-z_{\mathrm{II}}\right)}{z_{\mathrm{II}}}-\frac{\exp \left(-z_{\mathrm{I}}\right)}{z_{\mathrm{I}}}-\frac{1}{2}$

$\times\left[\frac{1+\left(z_{\mathrm{II}}-1\right)^{2}}{z_{\mathrm{II}}}-\frac{1+\left(z_{\mathrm{I}}-1\right)^{2}}{z_{\mathrm{I}}}\right]=0$

Systems (31), (32), and (33) togethei with condition (30) give the values of $z_{\mathbf{I}}, z_{\mathrm{II}} \epsilon^{\prime *}$, and $\Lambda$

The flame propagation model as described here gives the dimensionless flame spieading velocity $u /\left(\Omega \tilde{\lambda} / \bar{c}_{p}\right)^{1 / 2}$ in function of the heat of reaction $q_{r}$ and the mitial fuel or gas temperature $\Delta$, as shown in Fig 3(a) Howeves, to conclate these results with the values of $u / \nu$ given in Fig 2, it is necessary to know the law of var1ation of the heat of reaction with the mixture composition, represented by the mass fiaction $Y_{1 s 0}$ of fuel vapors in oxygen or in a given mixture of oxygen and nitrogen

There are no data avalable for the combustion of the fuel vapois of plexiglass in oxygen Therefore, a reasonable assumption will be made which consists in taking $q_{n} / \bar{c}_{p}=2000^{\circ} \mathrm{C}$ for the case of combustion in oxygen, for $Y_{1 s 0}=01$, which is probably close to the storchiometic condition, and then using a linear law of vartation of $q_{r} / c_{p}$ from that value down to zero for $Y_{1 s 0}=0$ 

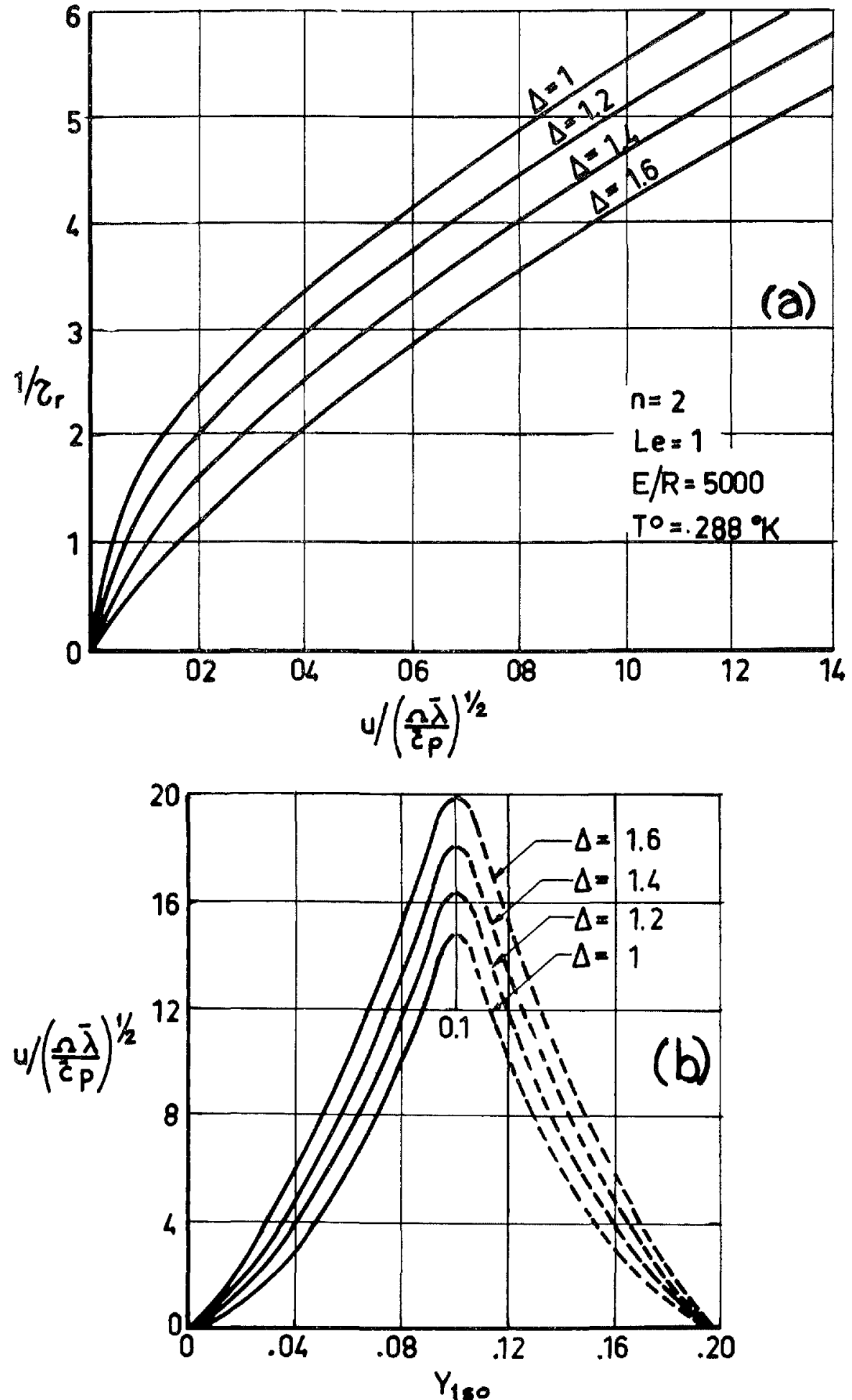

FIG. 3. (a) Flame spreading velocity as a function of the heat of reaction for several initial temperatures of the oxidizer at infinity, equal to the initial fuel temperature. (b) Flame spreading velocity calculated as a function of the fuel vapor mass fraction $Y_{1 s 0}$ for several values of the fuel temperature at infinity. 
$u /\left(\frac{\Omega \bar{\lambda}}{\bar{c} p}\right)^{1 / 2}$

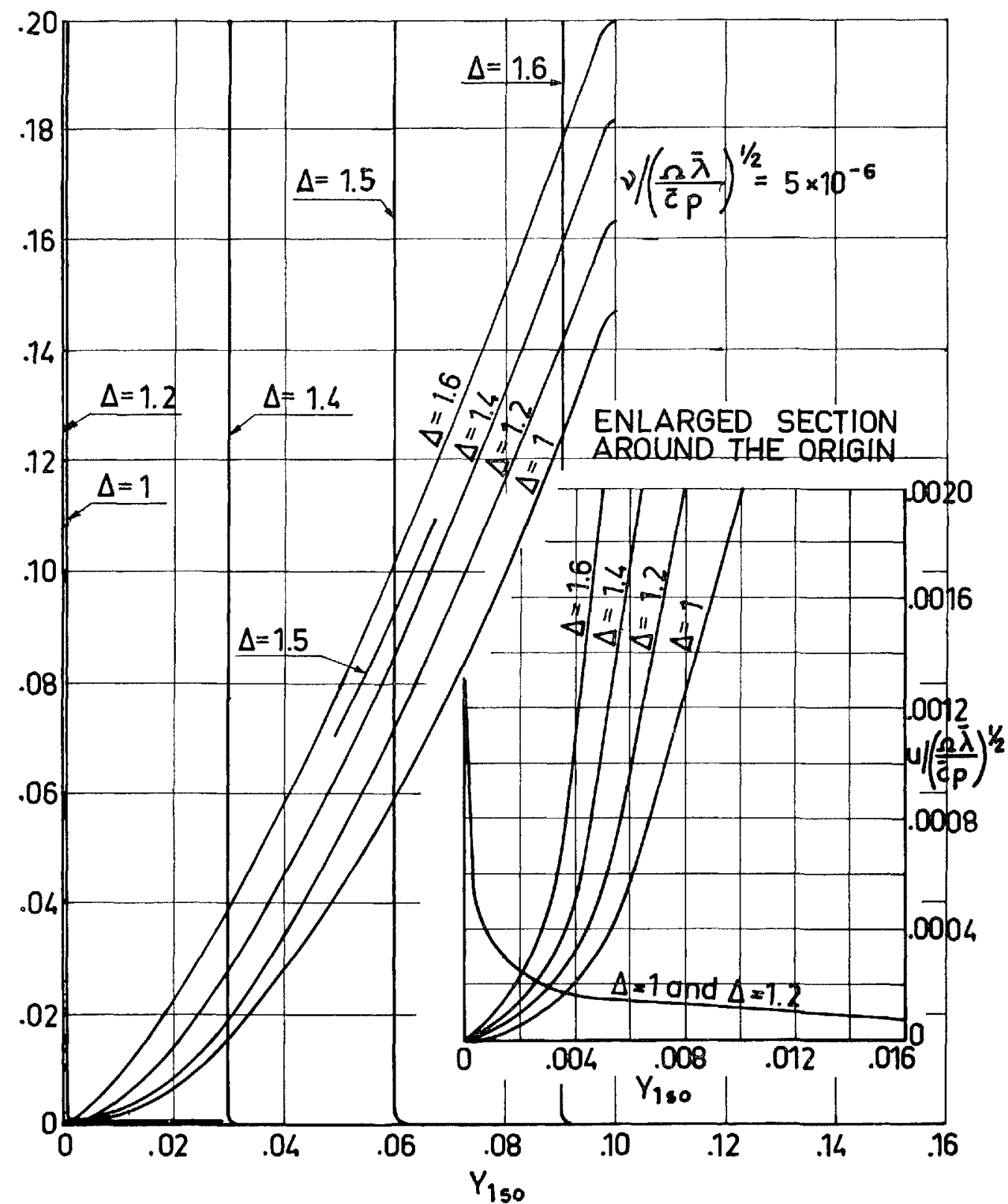

Fra. 4. Comparison of the assumed (Fig. 2) and calculated (Fig. 3b) spreading velocities.

and for $Y_{1, s 0}=0.2$. We have not acknowledged with this law of variation the lower and upper propagation limits for fuel vapors-oxygen mixtures.

With these linear laws of variation of $q_{r} / \vec{c}_{p}=$
$f\left(Y_{1 s 0}\right)$, the curves of Fig. $3(b)$ have been calculated, starting from the data of Fig. 3 (a). These curves give the dimensionless spreading velocity $u /\left(\bar{\Omega} \lambda / \bar{c}_{p}\right)^{1 / 2}$ in function of fuel vapor mass fraction $Y_{1 s 0}$. 


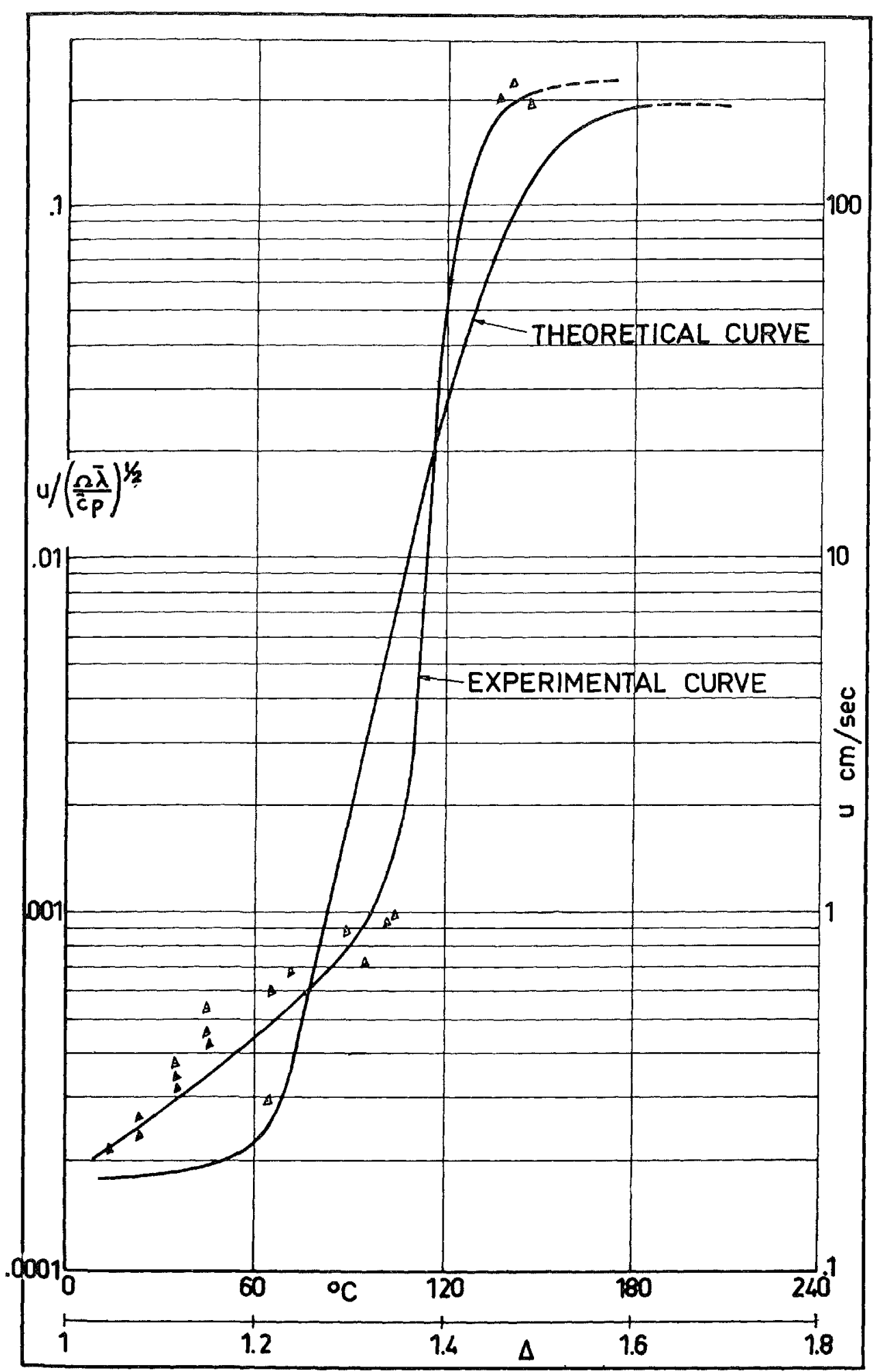

FIG. 5. Flame spreading velocities, calculated and measured over Plexiglass rods in oxygen at variable initial fuel temperature $(p=1 \mathrm{~atm})$. The qualitative agreement is satisfactory. 

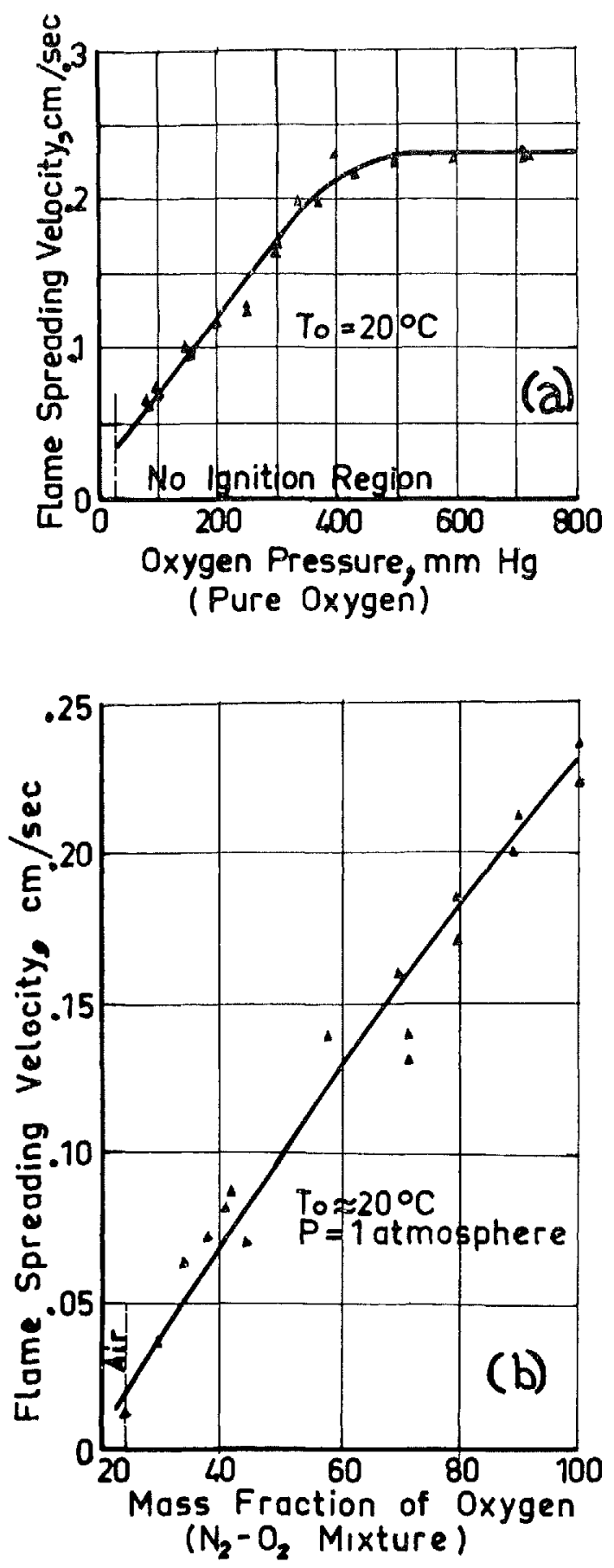

FIG. 6. (a) Flame spreading velocities over Plexiglass rods in oxygen at variable pressure $\left(T \sim 20^{\circ} \mathrm{C}\right)$ (b) Flame spreading velocities over Plexiglass rods in $\mathrm{N}_{2}-\mathrm{O}_{2}$ mixtures $(p=1$ atm, $T \sim 20^{\circ} \mathrm{C}$ ).

\section{Theoretical Results}

Theoretical rosults have been obtained giving the flame spreading velocity in function of the initial fuel temperature, by equating the values of $u / \nu$ given in Fig. 2 with those of $u /\left(\Omega \bar{\lambda} / \bar{c}_{p}\right)^{1 / 2}$ of Fig, $3(\mathrm{~b})$, as shown in Fig. 4.

To do this, a value must be given to parameter $\nu /\left(\Omega \bar{\lambda} / \bar{c}_{p}\right)^{1 / 2}$ and in the absense of data for the frequency factor $\Omega$, a value has been assigned to this parameter with the condition that the theoretical value of $u$ in the region of its maximum value will be of the same order of magnitude as the highest measured values of this velocity.

Figure 5 shows the resulting flame spreading: velocity as a function of the initial fuel temperature. In the region of low temperatures, the flame spreads very slowly, there is no significant combustible mixture ahead of the flame and heating of the fuel controls the spreading process. At a certain temperature, because of the exponential form of the vaporization functions, the fuel vapor mass fraction at the fuel surface increases rapidly, and the flame spreading velocity increases very sharply reaching values two or three orders of magnitude higher over a very short temperature interval. Actually, this temperature must be close to the "ignition temperature of the fuel," as usually defined. Above this temperature, a combustible mixture exists over the entire fuel surface and the flame propagates through this mixture, and heating of the fuel does not influence the process appreciably.

If the combustion properties of the fuel vapors and the oxidizers were known, it would be possible to calculate the influence of the mixture composition and gas pressure, at least approximately, neglecting possible variations of the flame size.

The flame temperature depends on mixture composition through the heat of reaction, and in this way the variation of the heat fluxes might be studied.

The influence of pressure is mainly exerted through the eigenvalue $\Lambda$, which is proportional to $\rho^{n}$. It also influences the heat transfer processes by conduction and convection, and if the pressure were reduced sufficiently, it would also influence chemical kinetics of the process and the flame temperature.

\section{Experimental Results}

An experimental program was carried out in which flame spreading velocities were measured over the surface of plastic fuels in a chamber filled with oxygen-nitrogen mixtures at pressures 
from 1 to $1 / 25 \mathrm{~atm}$ and at compositions ranging from air to pure oxygen.

The experiments were performed with plexiglass rods, held in a vertical position by a central wire which was electrically heated in order to control the initial temperature of the fuel. At temperatures above $90-100^{\circ}$, horizontal strips were also utilized because the rods became too soft to be held by the wire.

Flame spreading velocities as a function of the initial fuel temperature are shown in Fig. 5, where the theoretical curve has also been included. It may be scen that they are in satisfactory qualitative agrcement and that even the order of magnitude of the ratio of the values of the spreading velocities from the upper region to the lower region is very similar for both curves.

Figure 6 shows spreading velocities as functions of pressure and mixture composition. If these results are compared it may be seen that in pure oxygen, flame spreading velocities at pressures of the order of the corresponding partial pressure of the oxygen in arr are much higher than the spreading velocities in air.

\section{List of Principal Symbols}

$B=D / \nu \delta_{f}$ parameter

$C_{1}, C_{2}, K_{1}, K_{2}, K_{3}$ parameters and coefficients

$c_{f}$ specific heat of solid fuel

$\bar{c}_{p} \quad$ specific heat of mixture of gases

$\stackrel{\text { diffusion coefficient }}{D}$

$E \quad$ activation energy

Le $=\bar{\rho} \bar{D} \bar{c}_{p} / \bar{\lambda}$, Lewis-Semenov's number

$\dot{m}$ mass flow per unit area of reactants or reaction products

$\dot{m}_{f s} \quad$ mass flow of fuel evaporated per unit area and fuel surface

$n$ order of over-all reaction rate

$q_{1} \quad$ heat of vaporization

$q_{r} \quad$ heat of reaction

Pr Prandti number

$Q_{R}$ radiant heat

$R$ gas constant

Re Reynolds number

Sc Schmidt number

$T$ temperature

$T^{0} \quad$ reference temperature

$u$ spreading velocity

$x, y$ coordinatos

$Y$ mass fraction

$w \quad$ specific reaction rate

$z=\dot{m}_{12} \bar{C}_{p} / \bar{\lambda} x$ dimensionless coordinate

$\delta_{f}=\bar{\lambda}_{f} / \rho_{f} c_{f} \nu$ characteristic length

$\Delta \quad=T_{\infty} / T^{0}=1+\theta_{\infty}$ temperature parameter

$\epsilon \quad$ flux fraction of chemical species

$\eta \quad=y / \delta_{f}$ dimensionless coordinate $\xi=x / \delta_{f}$ dimensionless coordinate

$\lambda_{f}$ thermal conductivity of fuel

$\bar{\lambda}$ mean value of thermal conductivity of mixture of gases

$\Lambda$ eigenvalue of flame spreading system of equations

$\Omega \quad$ frequency factor

$\rho_{f} \quad$ fuel density

$\bar{\rho} \quad$ mean density of mixture of gases

$\theta \quad=\left(T-T^{0}\right) / T^{0}$ dimensionless fuel temperature

$\tau=\quad=\vec{c}_{p}\left(T-T_{\infty}\right) / q_{r}=\tau_{r} \theta$ dimensionless gas temperature

$\tau_{E}=E \bar{c}_{p} / q_{r} R$ parameter

$\tau_{r}=\bar{c}_{p} T_{\mathrm{co}} / q_{r}$ parameter

\section{Subscripts}

$f \quad$ solid fuel

$s$ at fuel surface

0 at origin

1 fuel vapors

2 oxidizer

$3 \quad$ reaction products

$\infty$ at infinity

\section{Superscripts}

1 derivative with respect to $z$

* at center of reaction zone

A CKNOWLINDGMUNTS

The r'esearch reported in this document has been sponsored by the Air Force Office of Scientific Research under Grant AF EOAR 67-44 through the European Office of Aerospace Research (OAR), United States Air Force.

The valuable advice given by Professor Amable Liñan is also acknowledged with appreciation.

\section{REFERENCES}

1. Anderson, H. E. And Rotheryesi, R. C.: Tenth Symposium (International) on Combustion, $\mathrm{p}$. 1009, The Combustion Institute, 1965.

2. Fons, W. L., Clemmints, H. B., and George, P. M.: Ninth Symposiam (International) on Combustion, p. 860, The Combustion Institute, 1963.

3. Emmons, H. W.: Tenth Symposium (Intenna(ional) on Combustion, p. 951, The Combustion Institute, 1965.

4. Hottel, H. C. and Williams, G. C.: Tenth Symposium (International) on Combustion, $\mathrm{p}$. 1253, The Combustion Institute, 1965.

5. Horowith, F. A.: Stevens Institute of Technology, Report, June 1965. 
6. Sanchez Tarifa, C. and Munoz Torralbo, A.: Eleventh Symposium (International) on Combustion, p. 533, The Combustion Institute, 1967.

7. Sanchez Tarita, C., Perge Del Notario, P. and Garcia Moreno, F.: Eighth Symposium
(International) on Combustion, p. 1035, Williams and Wilkins, 1962.

8. Perez Del Notario, P. and Sanchez Tarifa, C.: Ninth Symposium (International) on Combustion, p. 65, Academic Press, 1963.

\section{COMMENTS}

A. F. Roberts, Safety in Mines Research Establishment. In the regime where rate of spread of flame is controlled by preheating of the fuel, care must be exercised in applying your analysis to the spread of a flame across a liquid surface.

The analysis considers the conduction of heat along the fuel bed ahead of the flame. However in experiments with flames on liquid surfaces, I have observed well-defined convection currents in the liquid which serve to preheat the liquid ahead of the flame. The rate of heat transfer due to these currents has been estimated as 1000 times greater than that due to conduction, ${ }^{1}$

In the regime where an ignitable concentration of vapor exists above the fuel surface initially, my impression is that your analysis leads to a maximum value for the rate of spread close to the laminar burning velocity of the stoichiometric fuel/air mixture. However, in experiments with propanol $\left(\widehat{S}_{u} \simeq 0.45 \mathrm{~m} / \mathrm{sec}\right)$, I measured rates of spread up to $2 \mathrm{~m} / \mathrm{sec}$, which is approximately the velocity at which the hot products of combustion leave the flamefront, i.e., $\left(S_{u} \cdot T_{b}\right) / T_{u}$, although this may be a coincidence.

\section{REFERENCE}

1. Roberts, A. F.: Ph.D, thesis, University of London, 1959.

R. F. McAlevy III, Stevens Institute of Technology. The independent measurement of flame spreading velocity $(V)$ and distance along the surface affected by the spreading flame $(\delta)$, that has been made in my laboratory, might be useful in testing the validity of the particular means of nondimensionalizing the conservation equations employed by the authors. For example, it appears that the product $V \delta$ must equal the thermal diffusivity for the approach to be valid, and this could be checked quite readily.

W. A. Sirignano, Princeton University. I cannot agree with the statement that your flamespreading model applies to liquids as well as solids. For a variety of reasons, motion in the liquid can occur, modifying thermal-transfer processes and the spreading rate which destroys the model. For example, convection occurs since the heat-transfer and vaporization rates at the surface are not uniform. Also, if the liquid expands near the flame, gravity causes a flow along the surface. Another possibility is the effect of surface tension which is variable along the surface and can, therefore, produce motion along the surface.

C. Sanchez Tarifa, INTA, Madrid. We agree, indeed, that the processes of flame spreading over the surface of liquid or solid fuels might be different. However, the essential phenomenaheating of the fuel by the flame, fuel vaporization and mixing with the oxidizer, and flame propagation through this combustible mixture - are the same in both cases.

The effects mentioned by Prof. Sirignano probably are of secondary importance as compared with other effects disregarded in the model, such as the existence of tridimensional conditions and free-convection effects over the fuel surface. 